\title{
Measurement of quantum noise in a single-electron transistor near the quantum limit
}

\author{
W. W. Xue ${ }^{1 \star}$, Z. Ji ${ }^{2 \star}$, Feng Pan ${ }^{1}$, Joel Stettenheim ${ }^{1}$, M. P. Blencowe' ${ }^{1}$ and A. J. Rimberg' ${ }^{1 \dagger}$
}

Quantum measurement has challenged physicists for almost a century. Classically, there is no lower bound on the noise a measurement may add. Quantum mechanically, however, measuring a system necessarily perturbs it. When applied to electrical amplifiers, this means that improved sensitivity requires increased backaction that itself contributes noise. The result is a strict quantum limit on added amplifier noise ${ }^{1-6}$. To approach this limit, a quantum-limited amplifier must possess an ideal balance between sensitivity and backaction; furthermore, its noise must dominate that of subsequent classical amplifiers ${ }^{7}$. Here, we report the first complete and quantitative measurement of the quantum noise of a superconducting single-electron transistor (S-SET) near a double Cooper-pair resonance predicted to have the right combination of sensitivity and backaction ${ }^{8}$. A simultaneous measurement of our S-SET's charge sensitivity indicates that it operates within a factor of $\mathbf{3 . 6}$ of the quantum limit, a fourfold improvement over the nearest comparable results ${ }^{9}$.

The two mesoscopic devices most commonly used to electrically measure spin- and charge-based quantum systems are the singleelectron transistor (SET) and quantum point contact (QPC). These devices operate according to the same scheme: the electrometer is biased by a source-drain voltage $V_{\text {sd }}$ and the current $I$ through it is measured. Motion of charges near the electrometer causes its differential conductance $G_{\mathrm{d}}$ to change, resulting in changes in $I$. The ultimate sensitivity of an electrometer operated in this way is therefore set by the non-equilibrium current noise (shot noise) present in $I(t)$. The same current fluctuations also determine its backaction, and, therefore, its proximity to the quantum limit.

Classically, current noise is described by a spectral density $S_{I}{ }^{\text {sym }}(\omega)$ that is symmetric in frequency $\omega$. Quantum mechanically, however, we must distinguish between positive frequency noise, which transfers energy from a measured system to the electrometer, and negative frequency noise, which transfers energy from the electrometer to the measured system. A simple Fermi's golden rule calculation of, for example, an electrometer coupled to a qubit prepared in its ground state shows this ${ }^{10}$. The transition rate for the qubit to be promoted to its excited state is proportional to $S_{I}\left(-\omega_{0}\right)$, where $S_{I}(\omega)=\int_{-\infty}^{+\infty} \mathrm{d} t \mathrm{e}^{i \omega t}\langle I(t) I(0)\rangle$ is the unsymmetrized quantum noise spectrum of the electrometer current and $\hbar \omega_{0}$ is the separation in energy between the ground and excited states. Similarly, the rate at which a system prepared in its excited state decays to the ground state is given by $S_{I}\left(+\omega_{0}\right)$. To make a complete measurement of the quantum noise of an electrometer, one must obtain information regarding both $S_{I}\left(+\omega_{0}\right)$ and $S_{I}\left(-\omega_{0}\right)$.

Rather than couple our S-SET electrometer to a two-level system to carry out our quantum noise measurements, we instead couple it to another canonical quantum system, namely a harmonic oscillator consisting of an on-chip superconducting $L C$ resonator $^{11}$ as shown in Fig. 1a. This resonator serves both to impedance match the S-SET to the impedance $Z_{0}=50 \Omega$ of the measurement electronics ${ }^{12}$, and also to amplify its current noise so that it can be detected by a subsequent cryogenic amplifier.

In our S-SET/resonator system, the unsymmetrized shot noise of the S-SET at $\omega_{0}=1 / \sqrt{L C}$ is related to its probability to either emit energy to or absorb energy from the resonator. This enables a complete characterization of the noise. To see this, consider the Hamiltonian for the $L C$ resonator and S-SET given by

$$
\mathcal{H}=\frac{1}{2 L} \hat{\Phi}^{2}+\frac{1}{2 C} \hat{Q}^{2}-\hat{I}(t) \hat{\Phi}
$$

where $\hat{\Phi}$ is the flux in the inductor, $\hat{Q}$ is the charge on the capacitor and $\hat{I}(t)$ is the operator describing the noisy current flowing through the S-SET. This Hamiltonian is formally equivalent (see Supplementary Information) to one recently explored in the context of measuring the backaction of a charge detector on a nanomechanical resonator ${ }^{13-15}$. Assuming a large separation of timescales between fluctuations in $\hat{I}(t)$ and the response time of the $L C$ resonator, it can be shown rigorously that at the resonant frequency $\omega_{0}$ the S-SET can be viewed as an effective thermal bath, as illustrated in Fig. 1b, characterized by an effective temperature $T_{\mathrm{SET}}$ and a damping rate $\gamma_{\mathrm{SET}}$.

To make a complete noise measurement, it is not necessary to measure $S_{I}\left(+\omega_{0}\right)$ or $S_{I}\left(-\omega_{0}\right)$ separately, as has been done in other systems ${ }^{16-18}$. As long as two linearly independent combinations can be measured, complete noise information is obtained. This is how we proceed. Using equation (1) and the approach of refs $13-15$, it is simple to show that (for $\hbar \omega_{0}$ sufficiently small compared with $k_{\mathrm{B}} T_{\mathrm{SET}}$ )

$$
\begin{gathered}
S_{I}\left(\omega_{0}\right)+S_{I}\left(-\omega_{0}\right)=4 k_{\mathrm{B}} T_{\mathrm{SET}} C_{\mathrm{p}} \gamma_{\mathrm{SET}} \\
S_{I}\left(\omega_{0}\right)-S_{I}\left(-\omega_{0}\right)=2 \hbar \omega_{0} C_{\mathrm{p}} \gamma_{\mathrm{SET}}
\end{gathered}
$$

where $C_{\mathrm{p}}$ is the resonator capacitance. Note that $k_{\mathrm{B}} T_{\mathrm{SET}}$ can be significantly smaller than the energy of either the S-SET's physical temperature $k_{\mathrm{B}} T$ or its bias voltage $e V_{\text {sd }}$. Furthermore, it can be either positive or negative, as can $\gamma_{\mathrm{SET}}$, depending on whether absorption or emission, respectively, dominates the quantum noise.

Measuring the total symmetrized noise the S-SET injects into the resonator, while simultaneously measuring the rate $\gamma_{\mathrm{SET}}$ at which it damps the resonator modes therefore enables a complete measurement of the S-SET quantum noise without a separate measurement of either $S_{I}\left(+\omega_{0}\right)$ or $S_{I}\left(-\omega_{0}\right)$. A similar approach was used to investigate the backaction of an S-SET capacitively coupled to a nanomechanical resonator ${ }^{9}$. Our approach, in which

${ }^{1} 6127$ Wilder Laboratory, Department of Physics and Astronomy, Dartmouth College, Hanover, New Hampshire 03755, USA, ${ }^{2}$ Department of Physics and Astronomy, Rice University, Houston, Texas 77005, USA. ${ }^{\star}$ These authors contributed equally to this work. ${ }^{\dagger}$ e-mail: ajrimberg@dartmouth.edu. 

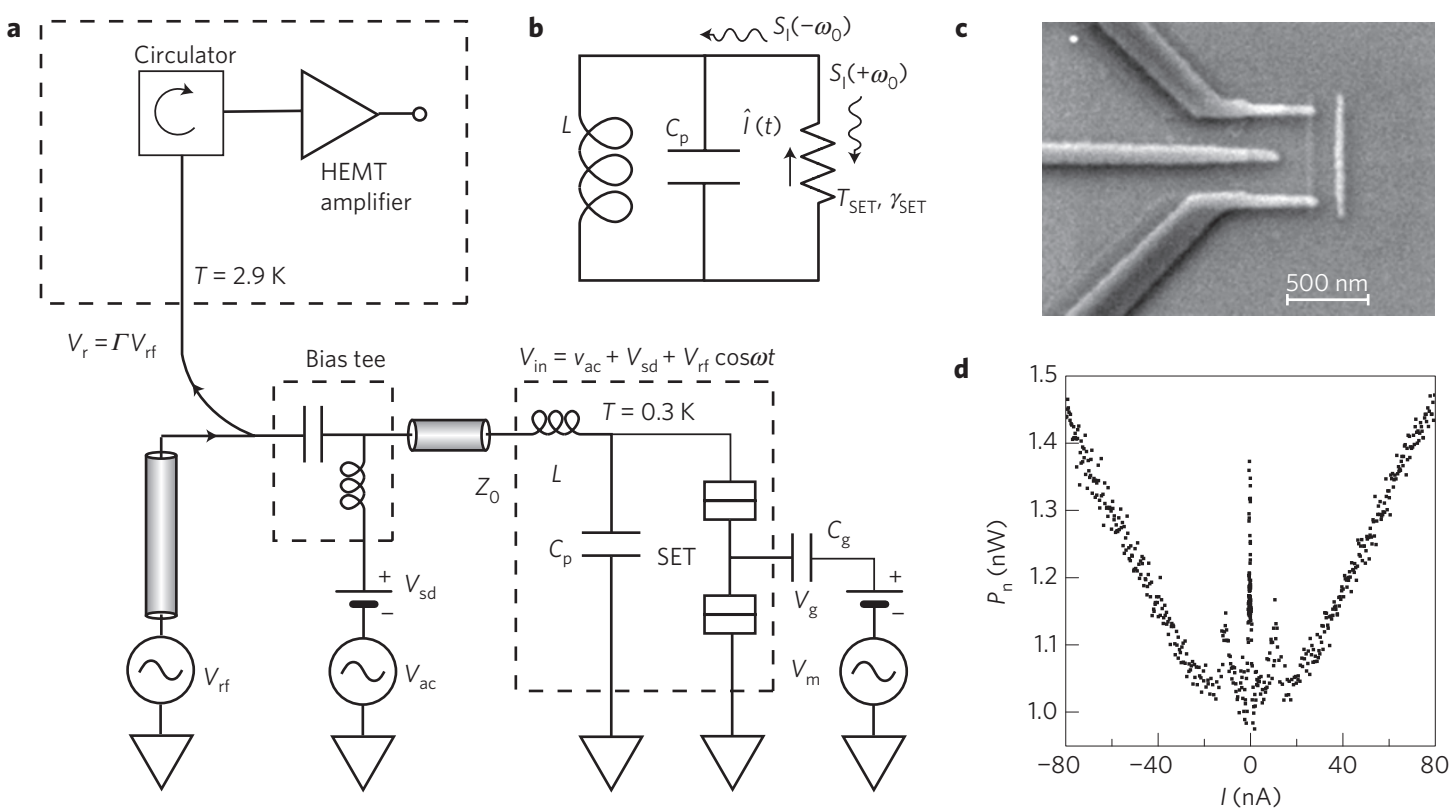

Figure 1 | Experimental method for quantum noise measurements. a, Measurement circuit, showing the sample and radiofrequency electronics, including a bias tee, circulator and cryogenic HEMT amplifier. b, Model for the S-SET/resonator, showing the S-SET as an effective bath with temperature $T_{\text {SET }}$ and damping rate $\gamma_{\text {SET }}$. The asymmetric current noise $S_{I}\left(+\omega_{0}\right)$ and $S_{I}\left(-\omega_{0}\right)$ is related to the probability of the S-SET absorbing or emitting a photon, as illustrated. c, Electron micrograph of a typical S-SET. d, Noise power $P_{n}$ at the output of the amplifier chain versus SET current $I$.

there is a direct electrical connection between the S-SET and a superconducting $L C$ resonator, is simpler to implement and enables a more accurate measurement of effective temperature and damping. Furthermore, the S-SET can easily be replaced by some other nanostructure such as graphene with interesting noise properties.

Our first step in characterizing the total quantum noise is to measure $\gamma_{\mathrm{SET}}=G_{\mathrm{d}} / C_{\mathrm{p}}$ by means of the S-SET's differential conductance $G_{\mathrm{d}}$. Although $G_{\mathrm{d}}$ is usually measured near d.c., extensive measurements of the reflection coefficient for waves incident on the resonator $\Gamma_{\text {in }}$ show ${ }^{11}$ that $G_{\mathrm{d}}$ accurately predicts $\Gamma_{\text {in }}$ and therefore $\gamma_{\text {SET }}$ at $\omega_{0}$. A plot of our measurements of differential conductance $G_{\mathrm{d}}$ versus $V_{\text {sd }}$ and island charge number $n_{\mathrm{g}}=V_{\mathrm{g}} C_{\mathrm{g}} / e$, where $V_{\mathrm{g}}$ is the gate voltage is shown in Fig. 2a. Interestingly, there are several points in the $V_{\mathrm{sd}}-n_{\mathrm{g}}$ plane at which $G_{\mathrm{d}}<0$. At these points, the S-SET exhibits negative differential conductivity (NDC). NDC is also clearly visible in Fig. $2 b$ as decreasing current with increasing bias just past the doubleJosephson quasiparticle (DJQP) current maximum. The NDC regions are associated with Cooper-pair resonances, occurring on the high-bias side of both the supercurrent and the DJQP features. In the DJQP subgap transport cycle, current flows by means of a combination of Cooper-pair and quasiparticle tunnelling ${ }^{19}$. This cycle appears as a peak in current near the intersection of two Cooper-pair resonances ${ }^{8,20}$, one for each junction in the S-SET, at $V_{\text {sd }}=2 E_{\mathrm{c}} / e$ as in Fig. $2 \mathrm{~b}$, where $E_{\mathrm{c}}=\mathrm{e}^{2} / 2 C_{\Sigma}$ is the S-SET charging energy.

When the S-SET is biased above the DJQP resonance (blue detuning), Cooper pairs must emit energy to tunnel. Similarly, when the S-SET is biased below the resonance (red detuning), Cooper pairs must absorb energy. As illustrated in Fig. 2d, because the S-SET's electromagnetic environment is dominated by the $L C$ resonator, most absorption (emission) will take the form of photon exchange with the tank circuit ${ }^{21}$. In terms of the picture of resonator damping given above, if $G_{\mathrm{d}}<0$ we expect both $\gamma_{\mathrm{SET}}<0$ and $\left|\Gamma_{\text {in }}\right|>1$. Physically, this negative damping corresponds to net emission of energy into the resonator by the S-SET. For the total symmetrized noise of the S-SET to remain positive (as it must), the S-SET effective temperature $T_{\mathrm{SET}}$ must also be negative in this region.
The second step in characterizing the total quantum noise of the S-SET is a measurement of $T_{\mathrm{SET}}$. This in turn first requires a measurement of the integrated SET shot noise $\mathcal{P}_{\mathrm{SET}}$, which has not previously been measured in the subgap regime. $\mathcal{P}_{\mathrm{SET}}\left(V_{\mathrm{sd}}, n_{\mathrm{g}}\right)$ at $300 \mathrm{mK}$ in the vicinity of the DJQP resonance is shown in Fig. 3a on a logarithmic scale. The noise is minimal for red detuning with respect to the DJQP, and maximal for blue detuning. We focused on the DJQP region for several reasons. First and foremost, an S-SET operated near the DJQP resonance has been predicted to possess the ideal balance of sensitivity and backaction needed to approach the quantum limit ${ }^{8}$. Second, near this cycle, the S-SET's quantum noise properties are expected to depend strongly on the SET bias in $V_{\text {sd }}$ and $n_{\mathrm{g}}$ with respect to this intersection ${ }^{8,14,15}$. Last, the charge sensitivity $\delta q$ of the S-SET is typically excellent here; charge sensitivity measurements as in Fig. $2 \mathrm{c}$ gave $\delta q \approx 1.7 \times 10^{-6} e / \sqrt{\mathrm{Hz}}$ for operation as a radiofrequency SET (ref. 12). It is interesting to note that in contrast, a normal-state SET biased near threshold is expected to operate far from the quantum limit ${ }^{22}$.

Our measurements of the S-SET noise characteristics show excellent correspondence with photon emission and absorption by the S-SET. We show this correspondence by measuring the reflection coefficient $\left|\Gamma_{\text {in }}\right|$ of the tank circuit over the same range of $V_{\mathrm{sd}}$ and $n_{\mathrm{g}}$, as in Fig. 3b. For most values of $V_{\mathrm{sd}}$ and $n_{\mathrm{g}}$, we found $\left|\Gamma_{\text {in }}\right|<1$, indicating net absorption by the S-SET. However, when the S-SET is blue detuned, there is a region for which $\left|\Gamma_{\text {in }}\right|>1$, indicating emission. Here the S-SET provides negative damping, returning more power to the resonator than is delivered by the radiofrequency excitation. Remarkably, therefore, as we measure $\left|\Gamma_{\text {in }}\right|>1$, we are directly measuring photon emission by Cooper pairs as they tunnel. Comparing this to the S-SET conductance in the same region as shown in Fig. 3d, we again see excellent correspondence. The region of negative damping corresponds exactly to the region of NDC. This is in accord with our expectation based both on the forms of $\gamma_{\mathrm{SET}}$ and $\Gamma_{\mathrm{in}}$, and with the more sophisticated quantum noise viewpoint of equations (2) and (3).

Having measured $G_{\mathrm{d}}$ and $\mathcal{P}_{\text {SET }}$, we now proceed to completely and quantitatively determine the quantum noise of our S-SET. As indicated above, we treat the S-SET as a thermal bath with 


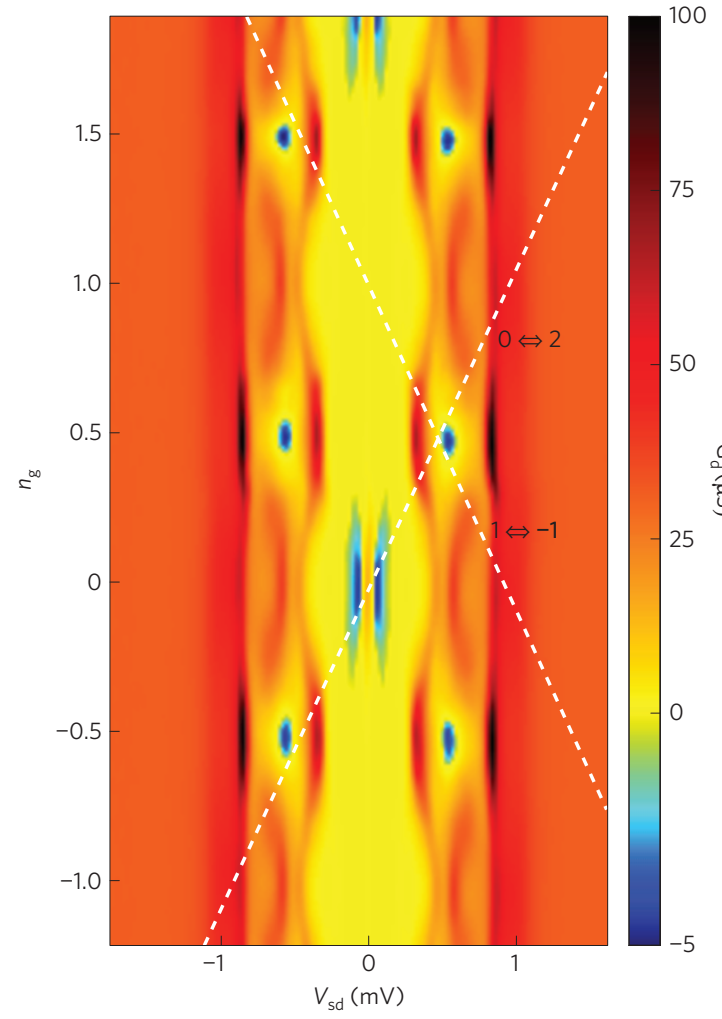

b

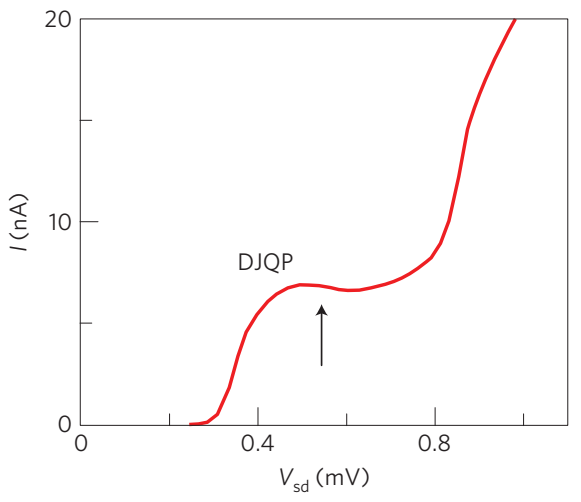

c

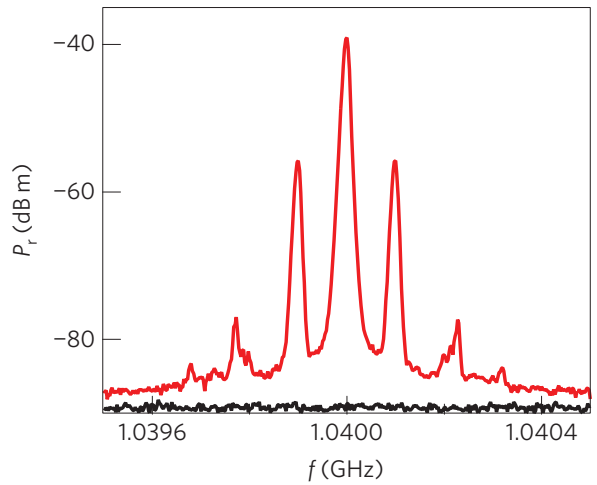

d

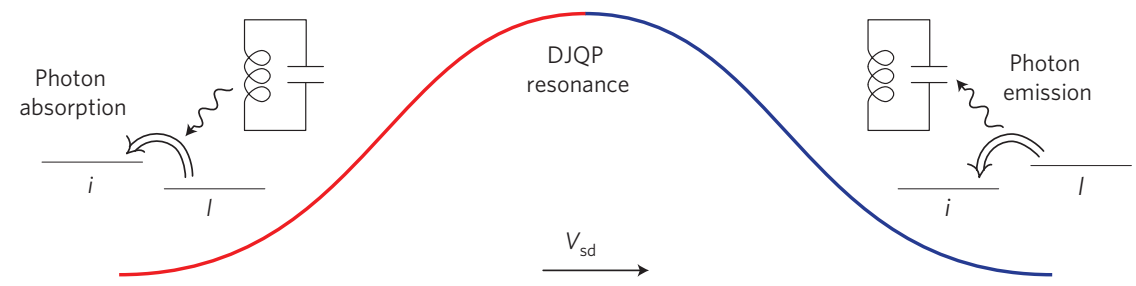

Figure 2 Subgap transport in the S-SET. a, $G_{d}$ for the S-SET versus $V_{s d}$ and $n_{g}$. NDC is visible for $V_{\text {sd }}$ and $n_{g}$ in the vicinity of the supercurrent and the DJQP cycle. Cooper-pair resonances $0 \Leftrightarrow 2$ and $1 \Leftrightarrow-1$ are shown as the dashed lines; the DJQP cycle occurs at their intersection. $\mathbf{b}, I-V$ characteristics of the S-SET for $n_{\mathrm{g}} \approx 0.5$, emphasizing the presence of NDC (arrow) on the high-bias side of the DJQP resonance where current decreases with increasing bias. c, Amplitude-modulated reflected power for a charge modulation of 0.01 at $100 \mathrm{kHz}$. The lower curve is the noise floor of the amplifier chain for $I=0$. d, DJQP cycle. When the S-SET is biased in $n_{\mathrm{g}}$ and $V_{\mathrm{sd}}$ so that Cooper pairs do not have enough energy to tunnel on or off the island (that is, the S-SET is biased to the left of both Cooper-pair resonance lines in $\mathbf{a}$ ), a photon must be absorbed from the resonator for tunnelling to occur. Similarly, when the S-SET is biased so that Cooper pairs have excess energy (to the right of both resonances in a), a photon must be emitted during tunnelling.

conductance $G_{\mathrm{d}}$ and available noise power $k_{\mathrm{B}} T_{\mathrm{SET}}$. It can be shown that $\mathcal{P}_{\mathrm{SET}}=4\left(\omega_{0} / \gamma_{\mathrm{T}}\right)^{2} Z_{0} G_{\mathrm{d}} k_{\mathrm{B}} T_{\mathrm{SET}} \Delta f\left(\right.$ where $\gamma_{\mathrm{T}}$ is the total damping rate of the resonator-see the Methods section), so that $T_{\mathrm{SET}}$ can be found from measurements of $\mathcal{P}_{\text {SET }}$, whereas $\gamma_{\text {SET }}$ can be determined directly from $G_{\mathrm{d}}$ through the relation $\gamma_{\mathrm{SET}}=G_{\mathrm{d}} / C_{\mathrm{p}}$. The resulting values of $\gamma_{\mathrm{SET}}$ and $T_{\mathrm{SET}}$ at $\omega_{0}$ versus $V_{\mathrm{sd}}$ and $n_{\mathrm{g}}$ near the DJQP for $300 \mathrm{mK}$ are shown in Fig. 4a,b. The tendency of the S-SET to either emit or absorb (as measured by $\gamma_{\mathrm{SET}}$ ) and its degree of asymmetry (as measured by $\left.T_{\mathrm{SET}} \propto\left(S_{I}\left(\omega_{0}\right)+S_{I}\left(-\omega_{0}\right)\right) /\left(S_{I}\left(\omega_{0}\right)-S_{I}\left(-\omega_{0}\right)\right)\right)$ vary strongly with $V_{\text {sd }}$ and $n_{\mathrm{g}}$. For blue detuning where Cooper pairs must give off energy, we observe both negative damping and a negative effective temperature. Although $T_{\mathrm{SET}}$ is large in some areas, for most bias points $T_{\mathrm{SET}} \lesssim 1 \mathrm{~K}$, making it smaller than $e V_{\text {sd }} / k_{\mathrm{B}}$ but still large enough that our assumption $k_{\mathrm{B}} T_{\mathrm{SET}} \gg \hbar \omega_{0}$ in equations (2) and (3) is still valid. For red detuning, where the S-SET is strongly absorbing, $T_{\mathrm{SET}}$ can be as low as $100 \pm 40 \mathrm{mK}$, less than the ambient temperature and indicating that the S-SET is capable of refrigeration. Although theoretical expressions for $\gamma_{\text {SET }}$ and $T_{\mathrm{SET}}$ near the DJQP exist ${ }^{14,15}$, they assume capacitive coupling of the S-SET to a resonator rather than our direct electrical connection, and also ignore higher-order tunnelling processes known $^{9,20}$ to be important for our relatively low-resistance S-SETs. Nonetheless, theory predicts a minimum $T_{\mathrm{SET}} \approx 250 \mathrm{mK}$ for an S-SET with our parameters, in reasonable agreement with our results. Finally, we prefer $T_{\text {SET }}$ and $\gamma_{\text {SET }}$ as a description of the S-SET quantum noise over the Fano factor because the latter is due only to fluctuations of the number of tunnelling electrons ${ }^{23}$. In our experiment, variations in $\mathcal{P}_{\mathrm{SET}}$ arising from electron number fluctuations are indistinguishable from those due to emission/absorption of photons.

We now estimate the measurement capability of our S-SET relative to the quantum limit. We imagine coupling the S-SET to some external device such as a quantum dot. The ratio of the time it takes the S-SET to measure the dot's charge state to the time it takes to dephase it must be greater than one. Quantitatively we express this condition in terms of the square root $\chi$ of this ratio given by $\chi=\sqrt{4\left(E_{\text {int }} / e\right)^{2} S_{Q}^{\text {sym }} S_{I}^{\text {sym }} /(\hbar \Delta I)^{2}} \geqslant 1$, where equality corresponds to the quantum limit. Here, $S_{Q}^{\text {sym }}$ and $S_{I}^{\text {sym }}$ are the symmetrized zerofrequency spectral densities of charge fluctuations on and current through the S-SET, $E_{\text {int }}$ describes its interaction with the measured system and $\Delta I$ is the change in S-SET current corresponding to a change in the system charge state $^{3}$ (see Supplementary Information). Using the current $I$ through the S-SET to estimate 
$\mathbf{a}$

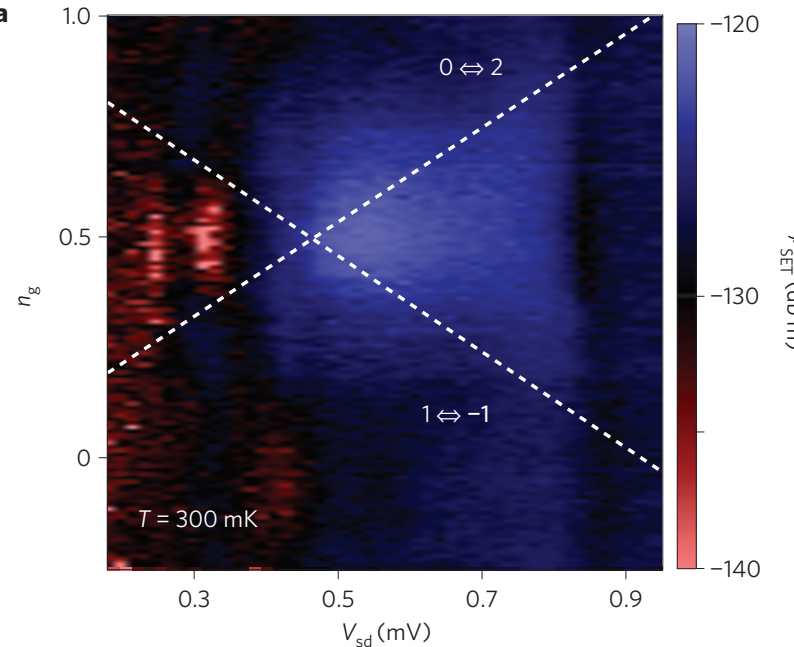

c

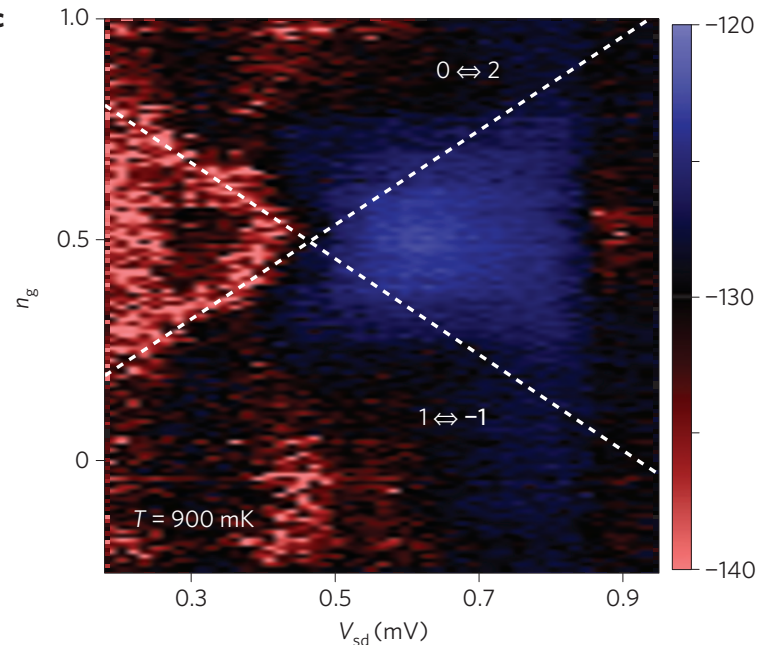

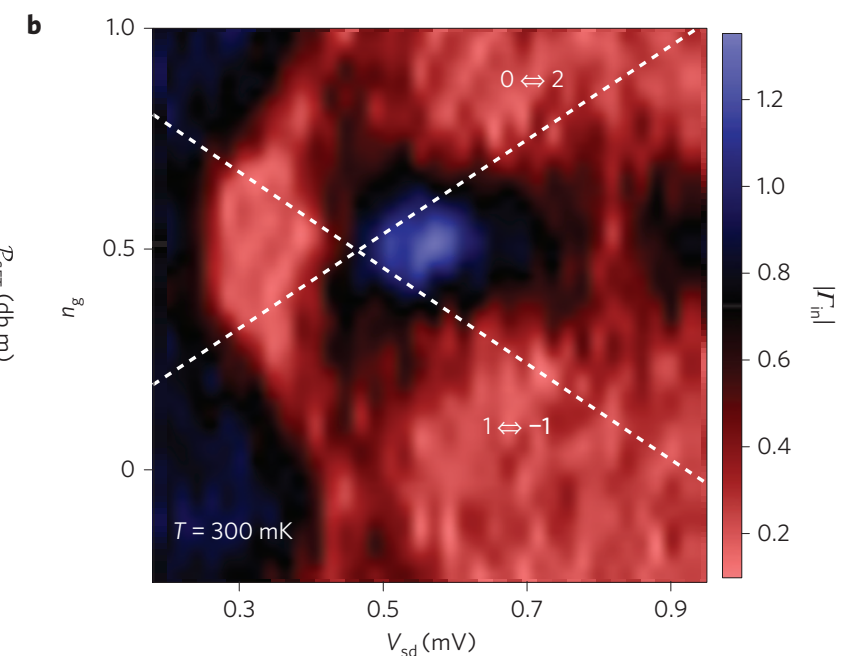

d

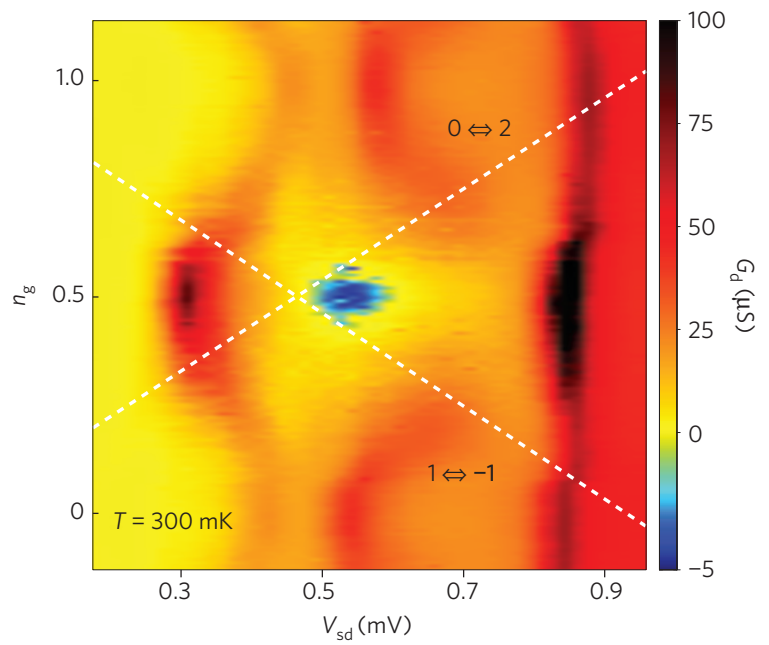

Figure 3 | Noise and reflected power measurements. a, $\mathcal{P}_{\mathrm{SET}}\left(V_{\mathrm{sd}}, n_{\mathrm{g}}\right)$ at $300 \mathrm{mK}$. Cooper-pair resonances are shown by the dashed lines, and the centre of the DJQP occurs at their intersection. Noise is maximal for blue detuning and minimal for red detuning. $\mathbf{b},\left|\Gamma_{\mathrm{in}}\right|\left(V_{\mathrm{sd}}, n_{\mathrm{g}}\right)$ at $300 \mathrm{mK}$. A small region for which $\left|\Gamma_{\text {in }}\right|>1$ exists for blue detuning. c, At $900 \mathrm{mK}, \mathcal{P}_{\mathrm{SET}}\left(V_{\mathrm{sd}}, n_{\mathrm{g}}\right)$ is smaller in the blue-detuned region (in agreement with a lessening of NDC there for higher temperature). The reduction of $\mathcal{P}_{\mathrm{SET}}$ in the red-detuned region is more pronounced, and tracks nearly exactly the Cooper-pair resonance lines. d, $G_{\mathrm{d}}\left(V_{\mathrm{sd}}, n_{\mathrm{g}}\right)$ at $300 \mathrm{mK}$. The region of NDC corresponds nearly exactly to that for which $\left|\Gamma_{\text {in }}\right|>1 \mathrm{in} \mathbf{b}$.

a

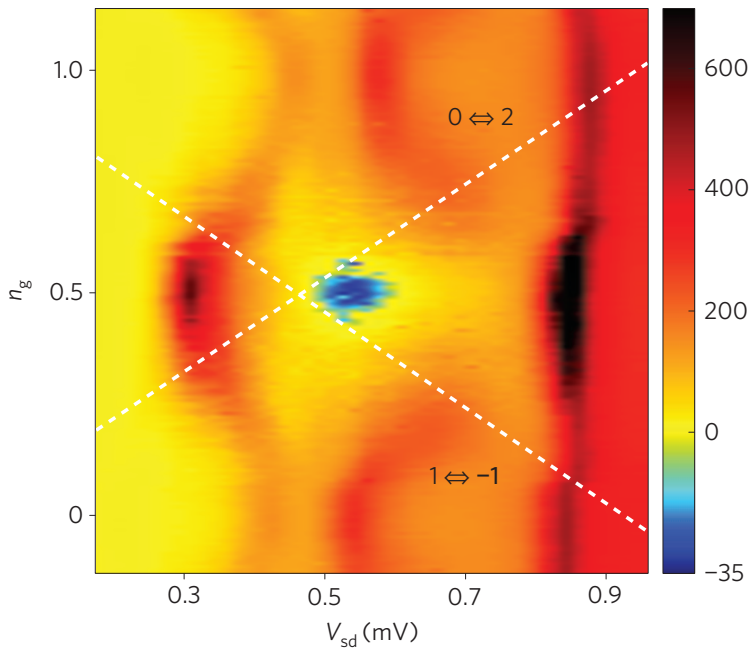

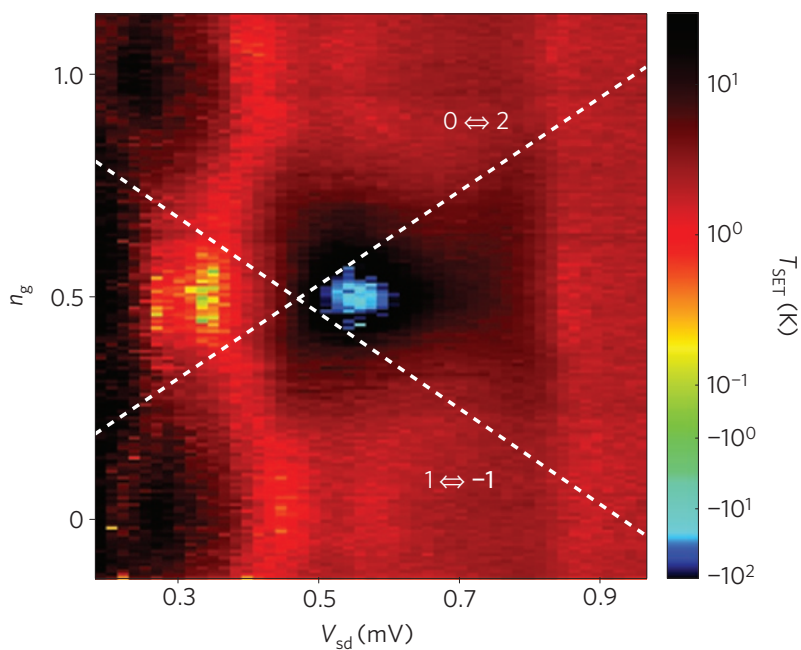

Figure 4 | Quantum noise of the S-SET. a, S-SET damping rate $\gamma_{S E T}$. b, S-SET effective temperature $T_{\text {SET }}$ at $\omega_{0}$. Together, these give a complete and quantitative description of the S-SET quantum noise. 
$S_{Q}^{\text {sym }} \approx 3 \mathrm{e}^{3} / 8 I$, we find $\chi \approx \sqrt{6 E_{\mathrm{c}}^{2} e(\delta q)^{2} / I \hbar^{2}}$ independent of the specifics of the dot and its coupling to the S-SET. This is as it should be: an amplifier's proximity to the quantum limit is an intrinsic property of the amplifier and does not depend on properties of the measured system. For typical currents $I \approx 5 \mathrm{nA}$ near the DJQP and neglecting the noise of the high-electron-mobility transistor (HEMT) amplifier, we find $\chi \approx 3.6$, indicating that our radiofrequency SET operates near the quantum limit. If amplifier noise is included, we find $\chi \approx 8$. These estimates each represent a fourfold improvement in $\chi$ over other results for both the S-SET (ref. 9), and a near-optimal normal-state SET (ref. 24). For the latter, we estimate an intrinsic $\chi \approx 20$. Note also that this approach probably overestimates $\chi$, because it ignores the presence of higherorder tunnelling processes that could bring the S-SET closer to the quantum limit ${ }^{25}$. In addition to its inherent interest in terms of quantum measurement, an S-SET charge sensor operating in the vicinity of the quantum limit has potentially broad implications in terms of its ability to measure a host of quantum systems.

\section{Methods}

All measurements were carried out in a ${ }^{3} \mathrm{He}$ refrigerator at its base temperature of $290 \mathrm{mK}$. The circulator and HEMT amplifier were at a temperature of $2.9 \mathrm{~K}$. A d.c. source-drain bias $V_{\text {sd }}$ and small a.c. voltage $v_{\text {ac }}$ were filtered and introduced to the high-frequency circuit by means of a bias tee. The input coaxial line included attenuation of $34 \mathrm{~dB}$. The data presented are for a representative sample. In all, five samples were measured, each producing similar results. (See Supplementary Information for details on sample parameters.) The resonator is a superconductin on-chip spiral ${ }^{11}$ for which internal losses are negligible, and can be fully described by its inductance $L \approx 169 \mathrm{nH}$ and its parasitic capacitance to ground $C_{\mathrm{p}}=0.14 \mathrm{pF}$, giving a resonant frequency $\omega_{0}=1.04 \mathrm{GHz}$. Its total damping rate $\gamma_{\mathrm{T}}$ is given by $\gamma_{\mathrm{T}}=\gamma_{0}+\gamma_{\mathrm{SET}}$, where $\gamma_{0}=Z_{0} / L$ is the damping due to the coupling to the feedline. The reflection coefficient $\Gamma_{\text {in }}$ for waves incident on the resonator can be written in terms of $\gamma_{0}$ and $\gamma_{\mathrm{SET}}$ as $\Gamma_{\mathrm{in}}=\left(\gamma_{\mathrm{SET}}-\gamma_{0}\right) /\left(\gamma_{\mathrm{SET}}+\gamma_{0}\right)$. On the basis of the simple model for the S-SET/resonator circuit in Fig. 1b, we expect $\gamma_{\mathrm{SET}}=G_{\mathrm{d}} / C_{\mathrm{p}}$. This relation, verified by extensive measurements of $\Gamma_{\mathrm{in}}$ versus $G_{\mathrm{d}}$, agrees with the expectation that $\omega_{0}$ is still in the low-frequency limit for the S-SET, because tunnelling events typically occur at a much higher rate (tens of gigahertz). To measure $\Gamma_{\text {in }}$, we applied a very small carrier wave $(-149 \mathrm{~dB} \mathrm{~m})$, measured the reflected power, and after accounting for the HEMT and circulator, computed $\Gamma_{\text {in }}$. To find the integrated noise $\mathcal{P}_{\text {SET }}$ we started by applying a d.c. current $I$ and measuring the total output noise power $P_{n}$ at $\omega_{0}$ in a bandwidth $\Delta f=5 \mathrm{MHz}$ at the output of the amplifier chain, as shown in Fig. 1a,d. The total output noise $P_{n}$ includes contributions from the S-SET, HEMT amplifier and circulator: $P_{n}=A\left(k_{\mathrm{B}} T_{\mathrm{HEMT}}+\left|\Gamma_{\text {in }}\right|^{2} k_{\mathrm{B}} T_{\text {circ }}+P_{\mathrm{SET}}(I)\right) \Delta f$, where $T_{\mathrm{HEMT}}$ and $T_{\text {circ }}$ are the HEMT and circulator noise temperatures, $P_{\mathrm{SET}}(I)$ is the spectral noise density of the SET referred to the HEMT input and $A$ is the total gain of the amplifier chain ${ }^{26}$. We use our noise power data to determine $A=61 \mathrm{~dB}, T_{\mathrm{HEMT}}=9.5 \mathrm{~K}$ and $T_{\text {circ }} \approx 2.9 \mathrm{~K}$, the last of which is in excellent agreement with the circulator's physical temperature (see Supplementary Information for details). This information enables us to extract $P_{\text {SET }}$ versus source-drain bias $V_{\text {sd }}$ and island charge number $n_{\mathrm{g}}$. The integrated SET noise referred to the input of the HEMT is given by $\mathcal{P}_{\mathrm{SET}}=P_{\mathrm{SET}} \Delta f$.

Received 22 January 2009; accepted 17 June 2009; published online 20 July 2009

\section{References}

1. Korotkov, A. N. Continuous quantum measurement of a double dot. Phys. Rev. B 60, 5737-5742 (1999).

2. Devoret, M. H. \& Schoelkopf, R. J. Amplifying quantum signals with the single-electron transistor. Nature 406, 1039-1046 (2000).

3. Makhlin, Yu., Schön, G. \& Shnirman, A. Quantum-state engineering with Josephson-junction devices. Rev. Mod. Phys. 73, 357-400 (2001).

4. Clerk, A. A., Girvin, S. M. \& Stone, A. D. Quantum-limited measurement and information in mesoscopic detectors. Phys. Rev. B 67, 165324 (2003).

5. Caves, C. M. Quantum limits on noise in linear amplifiers. Phys. Rev. D 26, 1817-1839 (1982).

6. Gardiner, C. W. \& Zoller, P. Quantum Noise (Springer, 2000).
7. Korotkov, A. N. Nonideal quantum detectors in Bayesian formalism. Phys. Rev. B 67, 235408 (2003).

8. Clerk, A. A., Girvin, S. M., Nguyen, A. K. \& Stone, A. D. Resonant Cooper pair tunneling: Quantum noise and measurement characteristics. Phys. Rev. Lett. 89, 176804 (2002)

9. Naik, A. et al. Cooling a nanomechanical resonator with quantum back-action. Nature 443, 193-196 (2006).

10. Schoelkopf, R. J., Clerk, A. A., Girvin, S. M., Lehnert, K. W. \& Devoret, M. H. in Quantum Noise in Mesoscopic Physics (ed. Nazarov, Yu. V.) 175-203 (Kluwer, 2003).

11. Xue, W. W. et al. On-chip matching networks for radio-frequency single-electron transistors. Appl. Phys. Lett. 91, 093511 (2007).

12. Schoelkopf, R. J., Wahlgren, P., Kozhevnikov, A. A., Delsing, P. \& Prober, D. E. The radio-frequency single-electron transistor (RF-SET): A fast and ultrasensitive electrometer. Science 280, 1238-1242 (1998).

13. Mozyrsky, D. \& Martin, I. Quantum-classical transition induced by electrical measurement. Phys. Rev. Lett. 89, 018301 (2002).

14. Blencowe, M. P., Imbers, J. \& Armour, A. D. Dynamics of a nanomechanical resonator coupled to a superconducting single-electron transistor. New J. Phys. 7, 236 (2005)

15. Clerk, A. A. \& Bennett, S. Quantum nanoelectromechanics with electrons, quasi-particles, and Cooper pairs: Effective bath descriptions and strong feedback effects. New J. Phys. 7, 238 (2005).

16. Deblock, R., Onac, E., Gurevich, L. \& Kouwenhoven, L. P. Detection of quantum noise from an electrically driven two-level system. Science 301, 203-206 (2003).

17. Billangeon, P., Pierre, F., Bouchiat, H. \& Deblock, R. Emission and absorption asymmetry in the quantum noise of a Josephson junction. Phys. Rev. Lett. 96, 136804 (2006).

18. Gustavsson, S. et al. Frequency-selective single photon detection using a double quantum dot. Phys. Rev. Lett. 99, 206804 (2007).

19. Fulton, T. A., Gammel, P. L., Bishop, D. J., Dunkleberger, L. N. \& Dolan, G. J. Observation of combined Josephson and charging effects in small tunnel junctions. Phys. Rev. Lett. 63, 1307-1310 (1989).

20. Thalakulam, M., Ji, Z. \& Rimberg, A. J. Sensitivity and linearity of superconducting radio-frequency single-electron transistors: Effects of quantum charge fluctuations. Phys. Rev. Lett. 93, 066804 (2004).

21. Lu, W., Maranowski, K. D. \& Rimberg, A. J. Charge transport processes in a superconducting single-electron transistor coupled to a microstrip transmission line. Phys. Rev. B 65, 060501 (2002).

22. Shnirman, A. \& Schön, G. Quantum measurements performed with a single-electron transistor. Phys. Rev. B 57, 15400-15407 (1998).

23. Choi, M. S., Plastina, F. \& Fazio, R. Shot noise for resonant Cooper pair tunneling. Phys. Rev. Lett. 87, 116601 (2001).

24. Brenning, H., Kafanov, S., Duty, T., Kubatkin, S. \& Delsing, P. An ultrasensitive radio frequency single electron transistor working up to $4.2 \mathrm{~K}$. J. Appl. Phys. 100, 114321 (2006)

25. Maassen van den Brink, A. Quantum-efficient charge detection using a single-electron transistor. Europhys. Lett. 58, 562-568 (2002).

26. Aassime, A., Gunnarsson, D., Bladh, K., Delsing, P. \& Schoelkopf, R. Radio-frequency single-electron transistor: Toward the shot noise limit. Appl. Phys. Lett. 79, 4031-4033 (2001).

\section{Acknowledgements}

This work was supported by the ARO under Agreement No. W911NF-06-1-0312, by the NSF under Grant Nos DMR-0804488 and DMR-0804477 and by the NSA, LPS and ARO under Agreement No. W911-NF-08-1-0482. We thank T. J. Gilheart, M. Bal and F. Chen for experimental assistance.

\section{Author contributions}

A.J.R. planned the experiment. W.W.X. and Z.J. fabricated the samples. W.W.X. carried out the measurements with assistance from Z.J., F.P. and J.S. M.P.B. proposed the method of analysis. W.W.X. and A.J.R. analysed the data. A.J.R. and J.S. wrote the paper with input from W.W.X. and M.P.B.

\section{Additional information}

Supplementary information accompanies this paper on www.nature.com/naturephysics. Reprints and permissions information is available online at http://npg.nature.com/ reprintsandpermissions. Correspondence and requests for materials should be addressed to A.J.R. 